\title{
Cerebrospinal Fluid (CSF) Concentration of Coenzyme Q10 in Clinical Practice, A Breaking News Finding in Humans
}

\author{
R.B. Singh ${ }^{1}$ and A. Naini ${ }^{2, *}$ \\ ${ }^{1}$ Halberg Hospital and Research Institute,Moradabad,India; \\ ${ }^{2}$ Department of Neurology, Columbia University Medical Center, New York, NY
}

\begin{abstract}
There is significant interest in coenzyme $\mathrm{Q}_{10}\left(\mathrm{CoQ}_{10}\right)$ as a potential treatment for neurodegenerative diseases and in its promise as a neuroprotectant. Experimental studies indicate that $\mathrm{CoQ}_{10}$ can protect against brain disorders by inhibiting neuronal damage. There is a need to demonstrate CSF levels of $\mathrm{CoQ}_{10}$ in humans.
\end{abstract}

\begin{abstract}
Subjects and Methods: After clearance from the hospital ethical committee, all of the subjects presenting with a suspicion of neurological diseases were asked to give written informed consent for lumber puncture for testing of CSF. CoQ ${ }_{10}$ concentration in CSF was examined by HPLC with electrochemical detection system at the Division of Molecular Neurogenetics, College of Physicians and Surgeons, New York,USA. Clinical and laboratory data were obtained from all of the patients $(n=38)$ to confirm clinical diagnosis. CAT scan of the head was done in all of the patients presenting with seizures and stroke. All CSF specimens were examined for cells, sugar and proteins and those suspected of being contaminated with blood were excluded from study.
\end{abstract}

Results: The age varied between 17 to 60 years and the number of males $(\mathrm{n}=16)$ were less than females $(\mathrm{n}=21)$. Patients were suffering from epileptic seizures $(n=19)$, tuberculous meningitis $(n=5)$ and stroke $(n=4)$. The rest of the 9 patients presented with headache of non-neurological condition with or without fever. The CSF concentration of CoQ $\mathrm{Q}_{10}$ was significantly lower among these subjects; mean $\pm \mathrm{SD}(0.59 \pm 0.12 \mathrm{ng} / \mathrm{ml})$, which appears to represent the normal level of $\mathrm{CoQ}_{10}$ in healthy subjects. The concentration of $\mathrm{CoQ}_{10}$ in CSF tended to be higher among patients with $\mathrm{TBM}(1.27 \pm 0.60$ $\mathrm{ng} / \mathrm{ml})$, seizures $(1.26 \pm 0.34 \mathrm{ng} / \mathrm{ml})$ and stroke $(0.83 \pm 0.20 \mathrm{ng} / \mathrm{ml})$ compared to subjects without neurological problem. These findings indicate that there is greater release of $\mathrm{CoQ}_{10}$ or increased synthesis in the tissues possibly to fight against oxidative stress caused by brain disorders.

Conclusions: This study shows that the level of $\mathrm{CoQ}_{10}$ in the csf is substantially lower than that in serum by three orders of magnitude and tends to be elevated under pathological conditions associated with brain injury. Further studies in a larger number of subjects are necessary to confirm our findings.

Key Words: Brain, ubiquinone, degenerative diseases, antioxidant, mitochondrial myopathy.

\section{INTRODUCTION}

Coenzyme $\mathrm{Q}_{10}\left(\mathrm{CoQ}_{10}\right)$, a lipid component of virtually all cell membranes, has several important biological functions. In the mitochondria, $\mathrm{CoQ}_{10}$ transfers electrons from complex I and complex II to complex III of respiratory chain. It is also an antioxidant [1], a membrane stabilizer [2], and in adipose tissues, it serves as a cofactor for uncoupling proteins [3].

$\mathrm{CoQ}_{10}$ deficiency has been observed in patients with Parkinsons disease, Huntington,s disease, tuberous sclerosis, motor neuron disease, and cerebellar ataxia, $\mathrm{CoQ}_{10}$ supplementation may be useful in these conditions [4-7]. $\mathrm{CoQ}_{10}$ deficiency has also been observed in patients receiving statins, which is known to cause myopathy and rhabdomyolysis [8-10]. Coenzyme $\mathrm{CoQ}_{10}$ administration increases, brain mitochondrial concentrations and exerts neuroprotectie effects $[11,12]$.

\footnotetext{
*Address correspondence to this author at the Molecular Neurogenetics, Columbia University, 1150 St. Nicholas Avenue, 3rd Floor, Rm 318, NY, NY 10032, USA; Tel: 212-305-2118; Fax: 212-305-3986;
}

E-mail: abn2@columbia.edu
$\mathrm{CoQ}_{10}$ is responsible for energy generation through the mitochondrial respiratory chain and because of its antioxidant properties, indirect support for pathogenetic role of $\mathrm{CoQ}_{10}$ deficiency is provided from data from patients with idiopathic,primary $\mathrm{CoQ}_{10}$ deficiency in mitochondrial myopathies [13-15]. These patients have a mitochondrial encephalomyopathy, most commonly presenting as a autosomal recessive spinocerebellar atrophy syndrome. A rarer myopathic varient combines central nervous system signs; ataxia,epilepsy,and mental retardation,with a mitochondrial myopathy dominated by recurrent rhabdomyolysis and myoglobinuria which are also an adverse effect of statin. A circadian rhythm in the coenzyme concentrations and a histochemical demonstration of a circadian rhythm of succinate dehydrogenase in rat pineal gland, and influence of $\mathrm{CoQ}_{10}$ addition has also been observed [16].

Naini and coworkers in 19 new patients with cerebellar ataxia established the existence of an ataxic syndrome due to primary $\mathrm{CoQ}_{10}$ deficiency and responsive to $\mathrm{CoQ}_{10}$ treatment [6]. Since all patients presented with cerebellar ataxia and cerebellar atrophy, this poses the possibility of a selective vulnerability of the cerebellam to $\mathrm{CoQ}_{10}$ deficiency. These workers found that in rats, cerebellum had the lowest level of 
$\mathrm{CoQ}_{10}$ and the level of $\mathrm{CoQ}_{10}$ was much higher in brain than in blood or visceral tissue, such as heart,liver or kidney indicating that it has vital role in brain function and degeneration [6]. However, there is lack of evidence, to demonstrate the cerebrospinal fluid levels of $\mathrm{CoQ}_{10}$ in normal subjects, compared to neurological degenerative diseases.

\section{SUBJECTS AND METHODS}

This is a case control study conducted in the Department of Internal Medicine, Halberg Hospital and Research Institute, Moradabad, India. It has a institutional ethic committee involving experts from other institutions and public. After clearance from the hospital ethic committee, all the subjects presenting with a suspicion of neurological diseases $(n=37)$ were asked to give written informed consent for lumber puncture for testing of CSF. CoQ 10 concentration in CSF was examined by HPLC with electrochemical detection after hexane extraction and purification. Briefly, CSF specimens were centrifuged at $3000 \mathrm{x} \mathrm{g}$ and $4{ }^{\circ} \mathrm{C}$ for $15 \mathrm{~min}$ and a $2-\mathrm{mL}$ aliquot was subjected to hexane extraction essentially as described by Lang e al. (1986). The HPLC system consisted of model 582 pump, model 542 autosampler, Coulochem II detector, 5011 Analytical Cell (E1 set at -600 Volts and E2 set at +450 Volts), and 5020 Guard Cell set at +800 Volts and positioned between autosampler and column. All the HPLC system components were from ESA (ESA, Inc., Shelmsford, MA). For the $\mathrm{CoQ}_{10}$ assay, the extracted $\mathrm{CoQ}_{10}$ was dissolved in $150 \mu \mathrm{L}$ of 1-propanol and a $50-\mu \mathrm{L}$ portion was directly injected onto the HPLC. The separation was achieved on a C18 reversed-phase column (Waters Corporation, Milford, MA) using a mobile phase containing $50 \mathrm{mM}$ sodium acetate in a mixture of ethanol,methanol, 2-propnol, and acetic acid $(50 \%, 47 \%, 1.5 \%$, and $1.5 \%$ respectively). Data acquisition and processing was performed utilizing EZ chrom HPLC software (Scientific Software Inc., Pleasanton, CA).

\section{Biochemical Method}

Clinical and laboratory data were obtained in all the patients $(n=38)$ to confirm the clinical diagnosis. CAT scan head was done in all the patients presenting with seizures and stroke. CSF was examined for cells, sugar and proteins in all the patients. Values were excluded in 5 subjects (11.7 to $26.9 \mathrm{ng} / \mathrm{ml}$ ) due to possible contamination with blood.

\section{RESULTS}

The age varied between 17 to 60 years and the number of males were fewer than females $(n=14$ vs 19$)$. Patients were suffering from epileptic seizures $(n=15)$, tuberculous meningitis $(n=4)$ and stroke $(n=4)$. Ten patients presented with headache of non-neurological condition with or without fever. The CSF concentration of $\mathrm{CoQ}_{10}$ was significantly lower among these subjects; mean $\pm \operatorname{SEM}(0.59 \pm 0.12, \mathrm{ng} / \mathrm{ml})$, which appears to be normal level of $\mathrm{CoQ}_{10}$ in healthy subjects. The concentration of $\mathrm{CoQ}_{10}$ in $\mathrm{CSF}$ was greater among patients with TBM $(1.27 \pm 0.60 \mathrm{ng} / \mathrm{ml})$, seizures $(1.26 \pm 0.34$ $\mathrm{ng} / \mathrm{ml})$ and stroke $(0.83 \pm 0.20 \mathrm{ng} / \mathrm{ml})$ compared to subjects without neurological problem. However, the differences were not significant (ANOVA P>0.05).

\section{DISCUSSION}

Here we report data presenting the levels of $\mathrm{CoQ}_{10}$ in control subjects (subjects with no known neurological ab- normalities) and in three groups of patients suffering some form of neurological disorder associated with brain injury. To our knowledge, this is the first report on the levels of $\mathrm{CoQ}_{10}$ in the CSF in these groups of patients, which compared to serum, is less by about three orders of magnitude. Such low levels of $\mathrm{CoQ}_{10}$ can only be reliably measured by HPLC with a very sensitive electrochemical detector system. The observation that the subjects, who presented with minimal brain disorders also had the lowest levels of $\mathrm{CoQ}_{10}$ in their CSF probably indicates that under pathological conditions associated with neurodegeneration, there is a rise in CSF $\mathrm{CoQ}_{10}$ as a result of either tissue damage and release from the cell or to increased synthesis as a cell response to free radical production caused by injury. In this context, it has recently been shown that increased levels of CoQ10 may protect cells from chemotherapy-induced oxidative stress [17].

In recent years, the possible beneficial effects of $\mathrm{CoQ}_{10}$ for the treatment of a wide variety of diseases, including disorders associated with neurodegeneration, has been investigated. In 1999, Singh and Singh used CoQ10 for the treatment of patients with motor neuron disease and polymyositis(18). However, in most of these studies, $\mathrm{CoQ}_{10}$ levels were measured in the plasma or the serum and few studies have assessed intracellular $\mathrm{CoQ}_{10}$ concentration in patients. In the plasma, $\mathrm{CoQ}_{10}$ is transported with the very low density lipoprotein (VLDL) fraction hence, it's levels are closely related to the plasma VLDL levels which can be affected by both the disease processes and by dietary factors. Moreover, whereas the cellular level of $\mathrm{CoQ}_{10}$ is dependent mostly on endogenous biosynthesis [18], the plasma level has been suggested as representing the equilibrium between $\mathrm{CoQ}_{10}$ absorption and synthesis [19]. Taken together, it appears that the plasma level of $\mathrm{CoQ}_{10}$ can not always be regarded as a true indicator of cellular levels. Therefore, in studies in which the cellular levels of $\mathrm{CoQ}_{10}$ and its changes under pathological conditions are investigated, tissues other than serum or plasma are more desirable. In this context, when the role of $\mathrm{CoQ}_{10}$ in diseases such as Parkinson's disease, Alzimer's disease, Huntuington's disease, or ALS is investigated, CSF is probably a true indicator of cellular level.

In conclusion, this is the first report of $\mathrm{CoQ}_{10}$ levels in the CSF as measured by highly sensitive HPLC with electrochemical detection system. These preliminary data indicate that (1) the level of CoQ in the CSF is less than in the serum by three orders of magnitude and (2) CSF levels tend to increase after injury. Obviously, with the availability of the methodology, further studies can be conducted to confirm and expand these preliminary findings.

\section{REFERENCES}

[1] Kagan V, Serbinova E, Packer L. Antioxidant effects of ubiquinones in microsomes and mitochondria are mediated by tocopherol recycling. Biochem Biophys Res Commun 1990; 169(3): 851-7.

[2] Turunen M, Olsson J, Dallner G. Metabolism and function of coenzyme Q. Biochim Biophys Acta 2004; 1660(1-2): 171-99.

[3] Echtay KS, Winkler E, Klingenberg M. Coenzyme Q is an obligatory cofactor for uncoupling protein function. Nature 2000; 408 (6812): 609-13.

[4] Group HsS. A randomized, placebo controlled trial of coenzyme Q10 ramacemide in Huntington's disease. Neurology 2001; 57: 375-376. 
[5] Lamperti C, et al. Cerebellar ataxia and coenzyme Q10 deficiency. Neurology 2003; 60(7): 1206-8.

[6] Naini A, et al. Primary coenzyme Q10 deficiency and the brain. Biofactors 2003; 18(1-4): 145-52.

[7] Singh RB, et al. Effect of coenzyme Q10 on risk of atherosclerosis in patients with recent myocardial infarction. Mol Cell Biochem 2003; 246(1-2): 75-82.

[8] Chiang CE, Pella D, Singh RB. Coenzyme Q10 and adverse effects of statins. J Nutr Environ Med 2004; 14: 17-28.

[9] Koumis T, et al. Strategies for the prevention and treatment of statin-induced myopathy: is there a role for ubiquinone supplementation? Am J Health Syst Pharm 2004; 61(5): 515-9.

[10] Rundek T, et al. Atorvastatin decreases the coenzyme Q10 level in the blood of patients at risk for cardiovascular disease and stroke. Arch Neurol 2004; 61(6): 889-92.

[11] Haas RH, et al. Low platelet mitochondrial complex I and complex II/III activity in early untreated Parkinson's disease. Ann Neurol 1995; 37(6): 714-22.

[12] Matthews RT, et al. Coenzyme Q10 administration increases brain mitochondrial concentrations and exerts neuroprotective effects. Proc Natl Acad Sci U S A 1998; 95(15): 8892-7.
[13] Di Giovanni S, et al. Coenzyme Q10 reverses pathological phenotype and reduces apoptosis in familial CoQ10 deficiency. Neurology $2001 ; 57(3): 515-8$.

[14] Musumeci $\mathrm{O}$, et al. Familial cerebellar ataxia with muscle coenzyme Q10 deficiency. Neurology 2001; 56(7): 849-55.

[15] Ogasahara S, et al. Muscle coenzyme Q deficiency in familial mitochondrial encephalomyopathy. Proc Natl Acad Sci U S A 1989; 86(7): 2379-82.

[16] Moller M, Hoyer PE. Histochemical demonstration of a circadian rhythm of succinate dehydrogenase in rat pineal gland. Influence of coenzyme Q10 addition. Histochemistry 1979; 59(4): 259-69.

[17] Brea-Calvo G, et al. Chemotherapy induces an increase in coenzyme Q10 levels in cancer cell lines. Free Radic Biol Med 2006; 40(8): 1293-302.

[18] Ernster L, Dallner G. Biochemical, physiological and medical aspects of ubiquinone function. Biochim Biophys Acta 1995; 1271(1): 195-204.

[19] Folkers K, Wolanivk A. Progress in biomedical research on coenzyme Q10. Drugs Exp Clin Res 1984; 10: 513-517.

(C) Singh and Naini; Licensee Bentham Open.

This is an open access article licensed under the terms of the Creative Commons Attribution Non-Commercial License (http://creativecommons.org/licenses/by$\mathrm{nc} / 3.0 /$ ) which permits unrestricted, non-commercial use, distribution and reproduction in any medium, provided the work is properly cited. 